\title{
Correction
}

\section{Correction: Loss of PGC- $1 \alpha$ in RPE induces mesenchymal transition and promotes retinal degeneration}

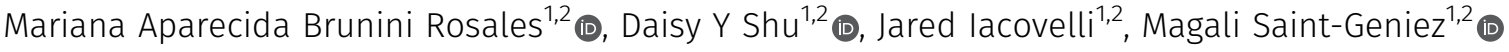 \\ ${ }^{1}$ Schepens Eye Research Institute of Massachusetts Eye and Ear, Boston, MA ${ }^{2}$ Department of Ophthalmology, Harvard Medical School, Boston, MA \\ DOI https://doi.org/10.26508/lsa.201900436 | Received 23 May \\ 2019 | Accepted 23 May 2019 | Published online 29 May 2019
}

See original article: LoSS of PGC-1 $\alpha$ in RPE induces mesenchymal transition and promotes retinal degeneration, 2(3), 2019.

Following publication, the authors realized that Table 1 inadvertently missed some primer sequences and contained primer sequences for genes not tested in this study. The table has now been replaced with the correct one. The authors regret any confusion this may have caused. Both the HTML and PDF versions of the article have been corrected. The corrected table is given below. 
Table 1. Primer sequences for qPCR.

\begin{tabular}{|c|c|c|c|}
\hline Gene symbol & Gene name & Forward sequence $\left(5^{\prime}-3^{\prime}\right)$ & Reverse sequence $\left(5^{\prime}-3^{\prime}\right)$ \\
\hline \multicolumn{4}{|c|}{ Human primers } \\
\hline ATG4D & Autophagy related 4D cysteine peptidase & CTCAACCCCGTGTATGTGC & TACAGTGAGTGTCGCGGTTT \\
\hline ATG9B & Autophagy related 9B & GCTACTGGGACATCCAGGTG & AAGAGGCGGGACTGCAC \\
\hline CAT & Catalase & ACTTTGAGGTCACACATGACATT & CTGAACCCGATTCTCCAGCA \\
\hline FIS1 & Mitochondrial fission 1 protein & TGACATCCGTAAAGGCATCG & CTTCTCGTATTCCTTGAGCCG \\
\hline GPX & Glutathione peroxidase 1 & CCAGTCGGTGTATGCCTTCTC & GAGGGACGCCACATTCTCG \\
\hline HMOX1 & Heme oxygenase 1 & GCCAGCAACAAAGTGCAAG & GAGTGTAAGGACCCATCGGA \\
\hline HPRT1 & Hypoxanthine phosphoribosyltransferase 1 & CCTGGCGTCGTGATTAGTGAT & AGACGTTCAGTCCTGTCCATAA \\
\hline LAMP1 & Lysosomal-associated membrane protein 1 & ACGTTACAGCGTCCAGCTCAT & TCTTTGGAGCTCGCATTGG \\
\hline MAP1LC3B & Microtubule associated protein 1 light chain 3 beta & GAGAAGACCTTCAAGCAGCG & TATCACCGGGATTTTGGTTG \\
\hline MCOLN1 & Mucolipin 1 & TTGCTCTCTGCCAGCGGTACTA & GCAGTCAGTAACCACCATCGGA \\
\hline MFN2 & Mitofusin 2 & ATGTGGCCCAACTCTAAGTG & CACAAACACATCAGCATCCAG \\
\hline PPARGC1A & $\begin{array}{l}\text { Peroxisome proliferator-activated receptor gamma, } \\
\text { coactivator } 1 \text { alpha }\end{array}$ & AGCCTCTTTGCCCAGATCTT & CTGATTGGTCACTGCACCAC \\
\hline PPARGC1B & $\begin{array}{l}\text { Peroxisome proliferator-activated receptor gamma, } \\
\text { coactivator } 1 \text { beta }\end{array}$ & CCACATCCTACCCAACATCAAG & CACAAGGCCGTTGACTTTTAGA \\
\hline POLG & DNA polymerase gamma, catalytic subunit & GAAGGACATTCGTGAGAACTTCC & GTGGGGACACCTCTCCAAG \\
\hline PPIA & Peptidylprolyl isomerase A & CAGACAAGGTCCCAAAGACAG & TTGCCATCCAACCACTCAGTC \\
\hline$P R C$ & PPARG related coactivator 1 (PPRC1) & GTGGTTGGGGAAGTCGAAG & TGACAAAGCCAGAATCACCC \\
\hline SOD1 & Superoxide dismutase 1 , soluble & AGGGCATCATCAATTTCGAGC & GCCCACCGTGTTTTCTGGA \\
\hline SOD2 & Superoxide dismutase 2, mitochondrial & CAGACCTGCCTTACGACTATGG & CGTTCAGGTTGTTCACGTAGG \\
\hline SIRT1 & Sirtuin 1 & TCAGTGTCATGGTTCCTTTGC & AATCTGCTCCTTTGCCACTCT \\
\hline SIRT3 & Sirtuin 3 & TGGAAAGCCTAGTGGAGCTTCTGGG & TGGGGGCAGCCATCATCCTATTTGT \\
\hline TFAM & Transcription factor a, mitochondrial & CCATATTTAAAGCTCAGAACCCAG & CTCCGCCCTATAAGCATCTTG \\
\hline TP53 & Tumor protein p53 & TCAACAAGATGTTTTGCCAACTG & ATGTGCTGTGACTGCTTGTAGATG \\
\hline TXN2 & Thioredoxin 2 & TGATGACCACACAGACCTCG & ATCCTTGATGCCCACAAACT \\
\hline TWIST1 & Twist family BHLH transcription factor 1 & CGGAGAAGCTGAGCAAGATT & TGGAGGACCTGGTAGAGGAA \\
\hline VIM & Vimentin & GAGAACTTTGCCGTTGAAGC & TCCAGCAGCTTCCTGTAGGT-3 \\
\hline WIPI1 & WD repeat domain, phosphoinositide interacting 1 & СCTCCTGGATATTCCTGCAA & GCACAATCTCCCCTGAAGTC \\
\hline ZEB1 & Zinc finger E-box binding homeobox 1 & AGTGATCCAGCCAAATGGAA & TTTTTGGGCGGTGTAGAATC \\
\hline ZEB2 & Zinc finger E-box binding homeobox 2 & AACAAGCCAATCCCAGGAG & GTTGGCAATACCGTCATCCT \\
\hline \multicolumn{4}{|c|}{ Mouse primers } \\
\hline Hprt1 & Hypoxanthine phosphoribosyltransferase 1 & TCAGTCAACGGGGGACATAAA & GGGGCTGTACTGCTTAACCAG \\
\hline Ppargc1a & $\begin{array}{l}\text { Peroxisome proliferator-activated receptor gamma, } \\
\text { coactivator } 1 \text { alpha }\end{array}$ & AGCCGTGACCACTGACAACGAG & GTCGCATGGTTCTGAGTGCTAAG \\
\hline Ppargc1b & $\begin{array}{l}\text { Peroxisome proliferator-activated receptor gamma, } \\
\text { coactivator } 1 \text { beta }\end{array}$ & CCCAGCGTCTGACGTGGACGAGC & CCTTCAGAGCGTCAGAGCTTGCTG \\
\hline Ppia & Peptidylprolyl isomerase A & GAGCTGTTTGCAGACAAAGTTC & CCCTGGCACATGAATCCTGG \\
\hline
\end{tabular}

License: This article is available under a Creative Commons License (Attribution 4.0 International, as described at https:// creativecommons.org/licenses/by/4.0/). 